\title{
Particle Sizing in Strongly Turbid Suspensions with the One-Beam Cross-Correlation Dynamic Light-Scattering Technique
}

Anthony J. Adorjan

James A. Lock

Cleveland State University, j.lock@csuohio.edu

Thomas W. Taylor

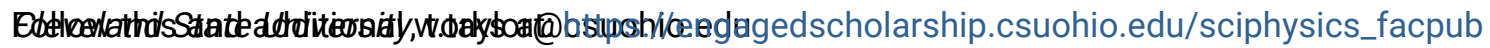

deatfaffthe Physics Commons

How does access to this work benefit you? Let us know!

Williamskeyesstatement

This paper was published in Applied Optics and is made available as an electronic reprint with

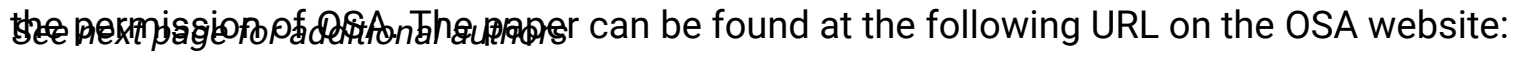
http://www.opticsinfobase.org/ao/abstract.cfm?URI=ao-38-15-3409. Systematic or multiple reproduction or distribution to multiple locations via electronic or other means is prohibited and is subject to penalties under law.

\section{Original Citation}

Adorjan, Anthony J., James A. Lock, Thomas W. Taylor, Padetha Tin, William V. Meyer, and Anthony E. Smart. "Particle Sizing in Strongly Turbid Suspensions with the One-Beam Cross-Correlation Dynamic Light-Scattering Technique." Applied Optics 38 (1999): 3409-3416.

\section{Repository Citation}

Adorjan, Anthony J.; Lock, James A.; Taylor, Thomas W.; Tin, Padetha; Meyer, William V.; and Smart, Anthony E., "Particle Sizing in Strongly Turbid Suspensions with the One-Beam Cross-Correlation Dynamic Light-Scattering Technique" (1999). Physics Faculty Publications. 88.

https://engagedscholarship.csuohio.edu/sciphysics_facpub/88

This Article is brought to you for free and open access by the Physics Department at EngagedScholarship@CSU. It has been accepted for inclusion in Physics Faculty Publications by an authorized administrator of EngagedScholarship@CSU. For more information, please contact library.es@csuohio.edu. 


\section{Authors}

Anthony J. Adorjan, James A. Lock, Thomas W. Taylor, Padetha Tin, William V. Meyer, and Anthony E. Smart

This article is available at EngagedScholarship@CSU: https://engagedscholarship.csuohio.edu/sciphysics_facpub/88 


\title{
Particle sizing in strongly turbid suspensions with the one-beam cross-correlation dynamic light-scattering technique
}

\author{
Anthony J. Adorjan, James A. Lock, Thomas W. Taylor, Padetha Tin, William V. Meyer, \\ and Anthony E. Smart
}

\begin{abstract}
The utility of the one-beam cross-correlation dynamic light-scattering system for sizing small particles in suspension was previously limited by its small-intensity signal-to-baseline ratio for strongly turbid suspensions. We describe three improvements in the optical system and sample cell that raise the ratio to a value comparable with that of other cross-correlation dynamic light-scattering systems. These improvements are (i) using a square cross-sectional sample cell to minimize the attenuation of the incident beam and singly scattered light, (ii) placing a $200-\mu \mathrm{m}$-wide slit between the sample cell and the detector fibers to mask off the region of weak single scattering and strong multiple scattering from the detectors' field of view, and (iii) aligning the center of the detectors' field of view with the region of strongest single scattering. We analyze a number of suspensions of polystyrene latex spheres with a diameter between 65 and $562 \mathrm{~nm}$ in water using this improved one-beam instrument and find that the measured radius is determined in a 2 -min data collection time to better than $\pm 10 \%$ for volume fractions of the suspended polystyrene latex spheres up to a few percent. (C) 1999 Optical Society of America

OCIS codes: $120.4640,290.5820,290.7050$.
\end{abstract}

\section{Introduction}

One of the many applications of dynamic light scattering is measuring the size of small noninteracting particles suspended in a liquid and undergoing Brownian motion. In dilute suspensions where the volume fraction of the suspended particles is $\phi \lesssim$ $10^{-5}$ and only single scattering occurs, the intensity correlation function $C(\tau)$ for both autocorrelation systems and cross-correlation systems consists of a constant baseline plus a decaying exponential in the delay time $\tau$. The decay rate is proportional to the

A. J. Adorjan is with the Department of Physics, Kent State University, Kent, Ohio 44242-0001. J. A. Lock and T. W. Taylor are with the Department of Physics, Cleveland State University, Cleveland, Ohio 44115-2440. J. A. Lock's e-mail address is jimandcarol@stratos.net. P. Tin is with the Ohio Aerospace Institute, NASA Lewis Research Center, Cleveland, Ohio 441353191. W. V. Meyer is with the National Center for Microgravity Research, NASA Lewis Research Center, Cleveland, Ohio 441353191. A. E. Smart is at 2857 Europa Drive, Costa Mesa, California 92626-3525.

Received 2 October 1998; revised manuscript received 3 February 1999 .

0003-6935/99/153409-08\$15.00/0

(C) 1999 Optical Society of America diffusion coefficient of the particles in the liquid, which in turn is inversely proportional to the particle radius. ${ }^{1}$ Larger volume fractions of either very small particles (e.g., a diameter of $\sim 10 \mathrm{~nm}$ ) or nearly index-matched particles give rise to negligible multiple scattering because of their very small scattering cross section and may also be analyzed relatively straightforwardly with dynamic light-scattering techniques. But a laser beam passing through a turbid suspension (e.g., $\phi>10^{-3}$ ) of particles, whose size is comparable to the wavelength of light and whose refractive index differs significantly from that of the liquid in which the particles are suspended (i.e., large, optically hard particles), undergoes significant multiple scattering. This produces a complicated nonexponential correlation function. ${ }^{2}$ To use dynamic light-scattering techniques as a nonintrusive method for sizing optically hard particles in strongly turbid suspensions (e.g., $\phi \approx 10^{-2}$ ), the contribution of multiple scattering to the measured intensity correlogram must either be suppressed or modeled and compensated for.

Many optical particle sizing instruments that use dynamic light scattering and that largely avoid contamination of the measured correlogram by multiple scattering have been demonstrated successfully. 
These include (1) autocorrelation systems using a tightly focused beam and a narrow field-of-view detector either in (a) side scattering ${ }^{3}$ or (b) backscattering, ${ }^{4-6}(2)$ cross-correlation systems using two counterpropagating laser beams of the same wavelength and two oppositely placed detectors whose narrow field of view is perpendicular to the beam paths, ${ }^{7-9}$ (3) the planar two-color crosscorrelation system ${ }^{10-12}$ that uses two different laser wavelengths and a scattering angle $\theta \neq 90^{\circ}$, (4) the three-dimensional two-color cross-correlation system, ${ }^{13-15}$ and (5) the one-beam cross-correlation system either (a) with ${ }^{16}$ or (b) without ${ }^{17}$ a gradientindex lens attached to the end of each of the two detector fibers. Systems (1a), (2)-(4), and (5a) possess the advantage of obtaining a large-intensity signal-to-baseline ratio $\beta$ (typically $0.80 \leqq \beta \lesssim 0.95$ for autocorrelation systems and $0.05 \leqq \beta \leqq 0.30$ for cross-correlation systems) when analyzing strongly turbid suspensions of optically hard particles. But these systems have the disadvantage of requiring sensitive and careful alignment because of the need to overlap the narrow field of view of the detector(s) with the tightly focused beam(s) in the sample cell. System (1b), on the other hand, enjoys the advantages of both a large $\beta$ and ease of alignment. System $(5 b)$, which is described in Section 2, has the advantages of a long depth of field and being easy to align because the field of view of the detectors is comparable to the width of the sample cell. But thus far it has had the disadvantage that, although the intensity signal-to-baseline ratio is $\beta \approx 0.2$ for polystyrene latex (PSL) spheres in water with $\phi \approx 10^{-4}$, the ratio falls to less than $\beta \approx 10^{-3}$ for these particles when $\phi \approx 10^{-2}$, thus requiring long run times and careful baseline fitting procedures when examining strongly turbid suspensions.

Our purpose in this paper is to describe three improvements to the one-beam cross-correlation system that increase its intensity signal-to-baseline ratio to $\beta$ $\approx 0.1$ for volume fractions of the suspended particles up to a few percent, while retaining its ease of alignment. These improvements make the one-beam cross-correlation system comparable in signal strength with other cross-correlation systems and greatly reduce the run time required to obtain a low-noise correlation function for strongly turbid suspensions. These improvements represent a necessary first step in making this instrument sufficiently robust to take it from the research laboratory to less forgiving industrial settings.

The body of this paper is organized as follows. In Section 2 we briefly review the one-beam system and describe the improvements in the optical system and sample cell. In Section 3 we present particle sizing results for a number of strongly turbid, nearly monodisperse suspensions of PSL spheres of diameter 65 $\mathrm{nm} \leq \mathrm{d} \leq 562 \mathrm{~nm}$ and volume fraction $0.001 \leq \phi \leq$ 0.05 in water. Last, in Section 4 we discuss our results and comment on some of the fundamental limitations of dynamic light-scattering systems for

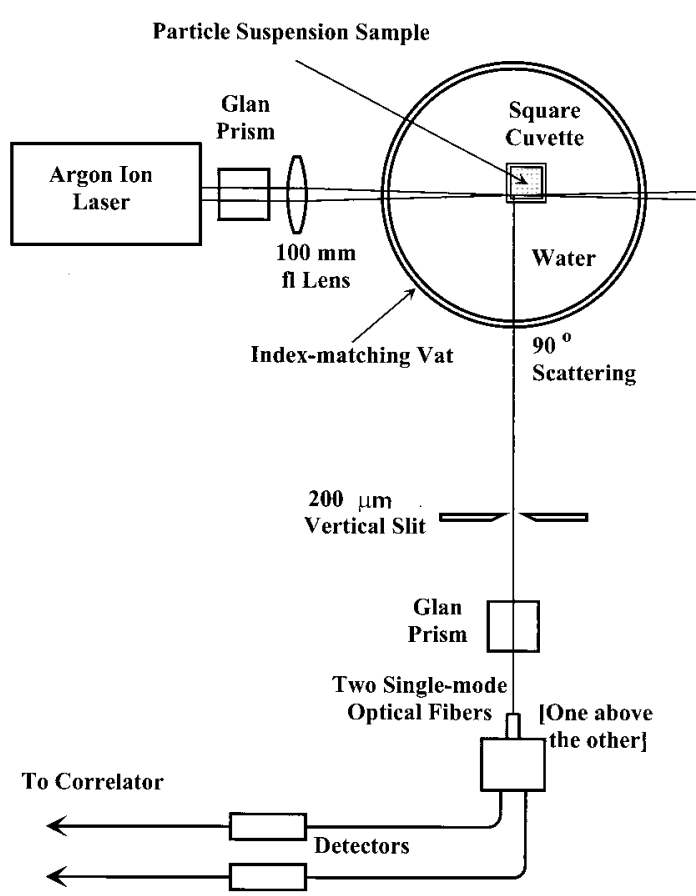

Fig. 1. One-beam cross-correlation dynamic light-scattering apparatus as seen from above. Light from an $\mathrm{Ar}^{+}$laser is focused by a $f=10$-cm lens, enters an index-matching vat filled with water, and is incident on a square cross-sectional sample cell. The light scattered at $\theta=90^{\circ}$ passes through a $200-\mu \mathrm{m}$-wide blocking slit and is coupled into two detector fibers that are separated vertically by $750 \mu \mathrm{m}$. Two Glan-Thompson prisms ensure that only the $V V$ polarization state of the incident and scattered light is detected. fl, focal length.

sizing optically hard particles in strongly turbid suspensions.

\section{One-Beam Cross-Correlation System}

\section{A. Overview of the Instrument}

The one-beam cross-correlation system with wide field-of-view detectors was described in detail in Refs. 17 and 18, and the version of it used here is illustrated schematically in Fig. 1. $\mathrm{An} \mathrm{Ar}^{+}$laser beam of wavelength $\lambda=514.5 \mathrm{~nm}$ is focused to a $44-\mu \mathrm{m}$ beam waist diameter by a $f=10-\mathrm{cm}$ lens and is incident on a sample cell containing small particles suspended in a liquid. Glan-Thompson prisms in the path of both the incident beam and the scattered light ensure that the incident and scattered electric fields are perpendicular to the scattering plane, the so-called $V V$ polarization state. Single scattering is confined to the beam volume within the cell, and multiple scattering visually appears as a much larger halo or glow surrounding the beam. Thus in the far zone, the singlescattering speckle size in the direction perpendicular to the beam is substantially larger than that for multiple scattering, whereas the single- and multiplescattering speckle sizes in the direction of the beam are comparable. ${ }^{19}$ At a scattering angle of $\theta=90^{\circ}$, two optical fibers, which have a single-mode cutoff of $\lambda=600 \mathrm{~nm}$, a numerical aperture of 0.16 , and with- 
out gradient-index lenses attached are connected to detectors and provide the wide field of view described above. They are stacked perpendicular to the beam direction so that their separation is greater than the multiple-scattering speckle size but less than the single-scattering speckle size. As a result, the singly scattered light cross correlates whereas all but a few percent ${ }^{13,18}$ of the multiply scattered light does not, producing the desired multiple-scattering suppression. To reduce flare produced by scattering from the surfaces of the sample cell and to prevent the $q$ broadening of the measured correlogram that would have otherwise occurred because of the wide field of view of the detectors, the sample cell was placed in a water-filled glass-wall cylindrical indexmatching vat of $8.0-\mathrm{cm}$ inner diameter and $8.5 \mathrm{~cm}$ outer diameter. The detectors were placed just beyond the vat's paraxial focal line, $17.0 \mathrm{~cm}$ from the scattering volume to focus the light scattered at $90^{\circ}$ onto the detector fibers. The detector fibers were raised a few degrees above the horizontal until the best signal-to-noise ratio was obtained, further reducing flare.

The particles' hydrodynamic radius $a$ was obtained in Ref. 17 by fitting the measured intensity correlogram to the formula appropriate for monodisperse noninteracting particles in the infinite-dilution limit:

$$
C(\tau)=\left\langle I_{\text {total }}\right\rangle^{2}\left[1+\beta \exp \left(-k_{B} T q^{2} \tau / 3 \pi \eta a\right)\right],
$$

where $\left\langle I_{\text {total }}\right\rangle$ is the time average of the scattered light intensity coupled into each of the detector fibers, the scattered momentum transfer is $q=4 \pi n \sin (\theta / 2) / \lambda$, $n$ is the refractive index of the liquid $(n=1.333$ for the experiments described both here and in Ref. (17), $k_{B}$ is Boltzmann's constant, $T$ is the temperature of the sample in degrees Kelvin, and $\eta$ is the viscosity of the liquid. The intensity signal-to-baseline ratio $\beta$ for autocorrelation or cross-correlation systems can be written as ${ }^{14}$

$$
\beta=\beta_{\text {geometric }} \beta_{\text {beams }}\left(\left\langle I_{\text {single }}\right\rangle /\left\langle I_{\text {total }}\right\rangle\right)^{2},
$$

where $\left\langle I_{\text {single }}\right\rangle$ is the time-averaged single-scattered light intensity recorded by the detectors, $\left\langle I_{\text {multiple }}\right\rangle$ is the time-averaged multiple-scattered light intensity recorded, and $\left\langle I_{\text {total }}\right\rangle=\left\langle I_{\text {single }}\right\rangle+\left\langle I_{\text {multiple }}\right\rangle$. The factor $\beta_{\text {geometric }}$ in Eq. (2) is the only contribution to $\beta$ for an autocorrelation system. It depends on geometric considerations such as the degree of overlap of the beam(s) with the field of view of the detector(s) and the ratio of the detector cross-sectional area divided by the spatial coherence area of the scattered light. The factor $\beta_{\text {beams }}$ depends on the intensity difference of the two illuminating beams in two-beam crosscorrelation systems. The factor $\left(\left\langle I_{\text {single }}\right\rangle /\left\langle I_{\text {total }}\right\rangle\right)^{2}$ pertains to both one-beam and two-beam crosscorrelation systems. In addition, the intensity signal-to-baseline ratio is further reduced by noise in the laser and the detector(s).
B. Improvements in the Optical System and Sample Cell The refractive index of PSL spheres relative to water is $n_{\text {rel }}=1.2$. Thus $\phi \approx 0.01$ suspensions of these particles whose size is comparable with the wavelength of light produce much multiple scattering. In Ref. 17 we found that, although the intensity signalto-baseline ratio was $\beta \approx 0.2$ for a $\phi=2.0 \times 10^{-4}$ concentration of $d=107-\mathrm{nm}$ PSL spheres, it fell to $\beta$ $=0.7 \times 10^{-3}$ for $\phi=0.01$, and no cross-correlation signal was detected for $\phi>0.01$. The reason for the small value of $\beta$ when $\phi \approx 0.01$ was because the detectors' wide field of view received an overwhelmingly large amount of multiply scattered light with respect to the amount of singly scattered light received. This occurred for three reasons: (i) The experiments described in Ref. 17 used a 1.01-cmdiameter circular cross-sectional glass test tube as the sample cell, and the focused laser beam traversed the cell diameter. In moderately turbid suspensions, for singly scattered light to exit the sample cell and be detected, it had to travel of the order of a cell radius in the suspension and was attenuated along the way. (ii) For strongly turbid suspensions, singly scattered light was found to be strong near the front surface of the sample cell and rapidly diminished along the beam path because of the attenuation of the laser beam as it penetrated into the cell. The multiple-scattering halo, on the other hand, was visually observed to be bright over the entire cell length. The wide field of view of the detectors encompassed the region of strong single and multiple scattering near the front of the sample cell, as well as the region of greatly attenuated single scattering and strong multiple scattering near the middle and the back of the cell. (iii) The coupling efficiency of scattered light into the detector fibers is greatest near the center of the fibers' field of view. ${ }^{20}$ In the experiments described in Ref. 17, the index-matching vat and the cell were concentric. Thus the center of the detectors' field of view was aligned with the center of the sample cell where single scattering was strongly attenuated and where most of the detected signal was multiply scattered light.

These three mechanisms conspired to produce the small value for the ratio $\left(\left\langle I_{\text {single }}\right\rangle /\left\langle I_{\text {total }}\right\rangle\right)^{2}$ in Eq. (2) for strongly turbid suspensions and suggested the following changes in our experimental setup: (i) We now use a $1.0 \mathrm{~cm} \times 1.0 \mathrm{~cm}$ square cross-sectional quartz cuvette as the sample cell within the index-matching vat. The focused laser beam is now incident on the cuvette's front wall approximately $0.5 \mathrm{~mm}$ from the side wall closest to the detector fibers as in Fig. 1. Thus singly scattered light travels only a short distance on its way to the detectors in the sample cell with much reduced attenuation. (ii) We now place a blocking slit, $200 \mu \mathrm{m}$ wide and $2 \mathrm{~cm}$ high, approximately half way between the sample cell and the detector fibers so as to obscure all but $\sim 0.4 \mathrm{~mm}$ of the fibers' field of view at the sample cell. The slit axis is perpendicular to the beam direction, and the slit is positioned so that the unobscured portion of the fi- 
bers' field of view contains only the region of strong single scattering near the front surface of the cuvette. The presence of the slit does not affect the single- and multiple-scattering speckle sizes perpendicular to the laser beam direction because the spatial extent of the single- and multiple-scattering effective sources ${ }^{19}$ in that direction is unchanged by the presence of the slit. (iii) We now align the center of the fibers' field of view with the slit and the strong single-scattering region near the front of the cell so as to most efficiently couple singly scattered light into the optical fibers. Each of these three improvements has been used at various times in the past in various dynamic light-scattering systems. But in the experiments described here we quantify the improvement that each produces in the signal-to-baseline ratio of the one-beam cross-correlation instrument.

In Ref. 17 the two detector fibers were separated by $250 \mu \mathrm{m}$ perpendicular to the beam direction. For most of the experiments described here, the fiber separation was chosen as $750 \mu \mathrm{m}$ for the following reason. Near the front of the sample cell where single scattering is the strongest, the multiple-scattering halo quickly fans out around the laser beam, and the halo radius increases as the beam propagates deeper into the cell. The blocking slit is positioned so that the region just inside the front wall of the cell is the only portion of the cell visible to the fibers. Thus the multiple-scattering speckle size perpendicular to the laser beam, though still smaller than that of single scattering, is larger than it was for the experiments of Ref. 17. This requires a larger detector separation so that the separation remains larger than the multiple-scattering speckle size.

\section{Experimental Results}

\section{A. Improvement in the Signal-to-Baseline Ratio}

The PSL suspensions analyzed in the present set of experiments were obtained from Bangs Laboratories. They were diluted with distilled water to the desired concentration and were interrogated by the instrument within a few minutes after preparation. Various electrolytes, whose purpose would be to screen electrostatic interactions, were not added to the suspensions. The fact that the particle size was measured accurately for the lower-concentration samples here, and in the experiments described in Ref. 17 where the same sample preparation method was used, provides some evidence that long-range electrostatic interactions did not strongly affect the diffusion coefficient of the PSL spheres in suspension. In the present set of experiments, we examined volume fractions $\phi=0.001,0.002,0.005,0.01,0.02$, and 0.05 of PSL spheres with diameter $d=65,120,246$, and 562 $\mathrm{nm}$. For all the experiments reported here, the scattering angle was $\theta=90^{\circ}$, and the $V V$ polarization state of the incident and scattered light was detected. The $\mathrm{Ar}^{+}$laser power was $100 \mathrm{~mW}$, except for the 65-nm particles, where the power was increased to $200 \mathrm{~mW}$ to compensate for the much lower lightscattering intensity at $90^{\circ}$ of these particles. Specif- ically, for the four particle sizes considered here, 65, 120,246 , and $562 \mathrm{~nm}$, the $V V$-polarized singlescattering intensity at $90^{\circ}$ was calculated using Mie theory and is in the ratio 1:32:454:872. The data collection time was $2 \mathrm{~min}$. This led to correlograms possessing a moderate amount of statistical noise. But this time period was used nonetheless because longer collection times are often impractical in industrial settings, and it is our purpose here to assess the one-beam instrument's viability for such applications.

We already mentioned that in the experiments described in Ref. 17, cross-correlated scattering by a $\phi$ $=0.01$ concentration of $d=107-\mathrm{nm}$ PSL spheres in water produced an intensity signal-to-baseline ratio of $\beta=0.7 \times 10^{-3}$. In the present experiments, we first determined the improvement in $\beta$ obtained by using the square cross-sectional cuvette, rather than the circular cross-sectional test tube, in the indexmatching vat. The focused laser beam now entered the front wall of the cuvette near its side wall, and the center of the detectors' field of view was aligned with the portion of the sample cell just inside the front wall of the cuvette as in Fig. 1. Cross-correlated scattering with this experimental arrangement by a $\phi=$ 0.01 concentration of $d=120$-nm PSL spheres in water, but without the blocking slit, produced an intensity signal-to-baseline ratio of $\beta=0.020$. This represents an increase by a factor of approximately 30 over the value of $\beta$ for the circular cross-sectional sample cell, corresponding to a decrease in the amount of multiple scattering detected relative to single scattering by a factor of approximately 30 . The increase in $\beta$ and the resulting decrease in the ratio of multiple scattering relative to single scattering are actually somewhat greater than these values. This is because the PSL spheres of Ref. 17 had a diameter of $107 \mathrm{~nm}$, whereas the spheres of this experiment have a diameter of $120 \mathrm{~nm}$, and the multiple-scattering intensity increases as the size of the particles increases for a given volume fraction of particles in suspension.

Next we inserted the blocking slit into the optical path as in Fig. 1 and varied the unobscured portion of the detectors' field of view from the front to the back of the cell. The slit and the unobscured portion of the sample cell were aligned with the center of the fibers' field of view by moving the sample cell within the index-matching vat along the incident beam direction using a micropositioner. The resulting value of $\beta$ as a function of slit position is shown in Fig. 2 (solid circles) for a $\phi=0.01$ concentration of $d=$ 65-nm PSL spheres. The measured diameter of the $d=65$-nm spheres is also shown in Fig. 2 (open circles). At the front wall of the cell, the intensity signal-to-baseline ratio quickly grows to $\beta \approx 0.14$. As the unobscured portion of the detectors' field of view is moved toward the back of the sample cell, $\beta$ progressively decreases because of the attenuation of the beam and the corresponding reduction in detected singly scattered light relative to multiply scat- 


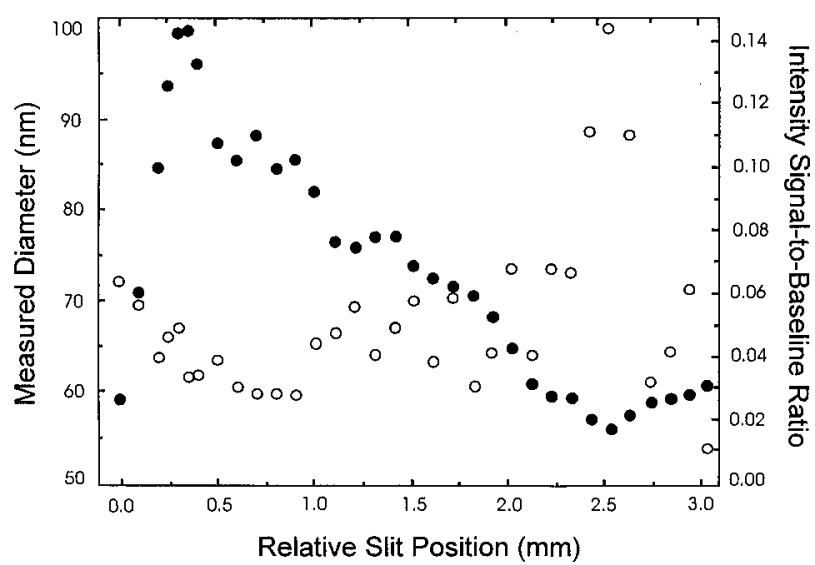

Fig. 2. Measured particle diameter (open circles) and intensity signal-to-baseline ratio $\beta$ (solid circles) as a function of the position of the blocking slit along the beam path in the sample cell. The front of the sample cell corresponds to the slit position $0.3 \mathrm{~mm}$. Only one 2-min measurement of the cross-correlation function was made at each slit position.

tered light. The single-scattering mean free path of light in the suspension is

$$
L_{s}=4 a / 3 \phi \epsilon_{\text {scatt }},
$$

where $\epsilon_{\text {scatt }}$ is the scattering efficiency of a single particle. For $d=65$-nm PSL spheres at $\phi=0.01$ in water, $\epsilon_{\text {scatt }}$ was calculated using Mie theory yielding $L_{s}=1.35 \mathrm{~mm}$. The intensity-to-baseline ratio in Fig. 2 was fit approximately by a decaying exponential with the decay distance $L_{s} \sim 1.7 \mathrm{~mm}$, in rough agreement with the calculated single-scattering mean free path.

The measured particle diameter was also found to be much less accurate deeper in the sample cell because of the poorer single-scattering statistics of the intensity cross-correlation function. But just past the front wall of the cuvette inside the cell is a region approximately $0.6 \mathrm{~mm}$ long where the measured diameter was found to be quite accurate and where $\beta \approx$ 0.10 indicating the region of relatively strong single scattering. This region is wider than the $\sim 0.4 \mathrm{~mm}$ length of the sample cell unobscured by the blocking slit and provides the optimal placement of the slit for the particle sizing experiments described below. Qualitatively similar results for $\beta$ as a function of slit position were obtained for a $\phi=0.01$ concentration of $d=246$-nm PSL spheres. No effort was made, however, to optimize $\beta$ by varying the width of the slit or its placement between the sample cell and the detector fibers.

As a final measurement of the improvement of $\beta$ for our one-beam system, we again considered the $\phi=$ 0.01 concentration of $d=120$-nm PSL spheres. Insertion of the blocking slit and positioning the unobscured portion of the detectors' field of view just inside the sample cell near its front wall produced an intensity signal-to-baseline ratio of $\beta=0.116$. This represents an increase in $\beta$ by a factor of approximately 6 from the value without the blocking slit and
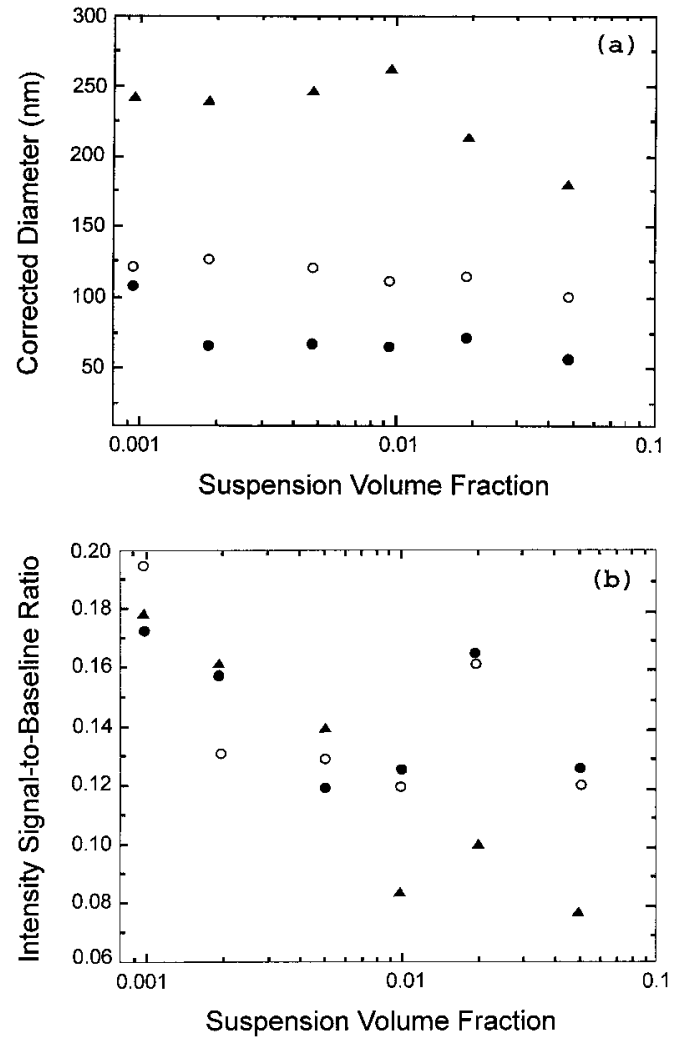

Fig. 3. (a) Measured particle diameter, corrected for hard-sphere particle interaction effects, as a function of the concentration of the suspended particles. The nominal particle diameter is $65 \mathrm{~nm}$ (solid circles), $120 \mathrm{~nm}$ (open circles), and $246 \mathrm{~nm}$ (triangles). Only one 2-min measurement of the cross-correlation function was made at each concentration. (b) Measured intensity signal-to-baseline ratio $\beta$ as a function of the concentration of the suspended particles. The particle nominal diameter is $65 \mathrm{~nm}$ (solid circles), 120 $\mathrm{nm}$ (open circles), and $246 \mathrm{~nm}$ (triangles). Only one 2-min measurement of the cross-correlation function was made at each concentration.

a factor of approximately 170 from that of the experiment of Ref. 17 using the circular cross-sectional sample cell. Similar improvements in $\beta$ that were due to the blocking slit were obtained for all particle sizes and volume fractions examined, with the improvement being somewhat larger for smaller concentrations.

\section{B. Particle Sizing Results}

With the detector fibers separated by $750 \mu \mathrm{m}$, the measured particle radii, corrected for volume exclusion and hyrodynamic particle interaction effects, and the intensity signal-to-baseline ratio for suspensions containing $d=65-, 120-$, or $246-\mathrm{nm}$ particles are shown as a function of volume fraction in Figs. 3(a) and 3(b), respectively. The particle radius and the intensity signal-to-baseline ratio in each case was determined by fitting the measured correlogram to Eq. (1) which assumes single scattering only, a monodisperse sample, and the absence of flare and the partial homodyning that it produces. Each data point in Fig. 3 (as well as in Fig. 2) corresponds to a 
single 2-min measurement of the cross-correlation function, as is characteristic of single-shot particle sizing measurements.

Presuming for the moment that multiple scattering has been completely suppressed and flare has been completely eliminated so that all the detected light corresponds to single scattering, particle interactions begin to affect the measured PSL sphere radius inferred from Eq. (1) at volume fractions larger than $\phi$ $\geq 0.01$. The four particle sizes used in the present experiments correspond to the argument $q a$ of the static structure factor having the value $0.75,1.38$, 2.83 , and 6.46. These values span the region from $q a \lesssim 2$ where particle interactions are dominated by collective diffusion mechanisms to $q a \gtrsim 2$ where particle interactions are dominated by self-diffusion mechanisms. ${ }^{4,21-23}$ We assumed that the PSL spheres suspended in water are adequately modeled as hard spheres, and we corrected the measured particle radius obtained from Eq. (1) for volume exclusion and hydrodynamic effects using the hydrodynamic model of Eqs. (92a)-(92e) of Ref. 23 (which is accurate for volume fractions of a few percent or less) and the Percus-Yevick hard-sphere structure function and pair correlation function. ${ }^{24}$ Figure 3(a) indicates that the corrected hydrodynamic radius of the PSL spheres was generally determined by our onebeam cross-correlation instrument to better than $\pm 10 \%$ for $\phi \leq 0.05$.

The larger than expected measured size of the $65-\mathrm{nm}$ particles at $\phi=0.001$ is probably due in part to their relatively weak single-scattering intensity at $90^{\circ}$ and the resulting relatively large statistical noise in the correlogram measured in a single 2-min interval. For the 562-nm particles, the detector fibers were spaced by $500 \mu \mathrm{m}$ because we could not obtain a clean, low-noise correlogram using a $750-\mu \mathrm{m}$ separation. The measured radii of the $562-\mathrm{nm}$ particles followed the same trends as for the 246-nm particles. But they are not plotted in Fig. 3 because we desired all the data shown in the figure to be obtained with the same instrumental geometry.

As can be seen in Fig. 3(a), the one-beam crosscorrelation technique somewhat undersized the 120and 246-nm particles for $\phi \geq 0.02$. One likely reason for this is the residual few-percent crosscorrelation of the detected multiply scattered light. ${ }^{13,18}$ Residual cross-correlated multiple scattering causes the correlation function to decay fast$\mathrm{er}^{2,4}$ as a function of $\tau$ than if single scattering alone were present. As a result, the measured particle radius obtained using Eq. (1) in the presence of weakly cross-correlated multiple scattering is underestimated. Other possible reasons for undersizing the larger particles at the higher concentrations include the effects of incoherent and coherent flare. The former adds noise to the correlogram and the latter acts as a local oscillator, which also effectively undersizes the particles if Eq. (1) is used for the analysis. Experiments whose purpose is to assess the importance of flare and partial homodyning are now in progress, and preliminary results for autocorrelation systems have been published in Ref. 25 .

One measure of the amount of multiply scattered light present is given by a comparison between the size of the sample cell and the single-scattering mean free path. The longest single-scattering mean free path for the suspensions examined here is $L_{s}=13.5$ $\mathrm{mm}$ corresponding to the $d=65 \mathrm{~nm}$ and $\phi=0.001$ sample, which is comparable with the length of the sample cell. As a result, all the suspensions examined here visually appear milky white to the naked eye. The noticeably low measured sizes in Fig. 3(a) for $d=120 \mathrm{~nm}$ and $\phi=0.05, d=246 \mathrm{~nm}$ and $\phi=$ 0.02 , and $d=246 \mathrm{~nm}$ and $\phi=0.05$ correspond to $L_{s}$ $=51,34$, and $14 \mu \mathrm{m}$, respectively. Thus the improvements to the one-beam instrument described here permit the sizing of PSL spheres in water in a 2 -min time interval to $\sim 10 \%$ accuracy until $L_{s}$ is an order of magnitude less than the $400 \mu \mathrm{m}$ length of the sample cell unobscured by the blocking slit. Although a $10 \%$ error is not sufficiently accurate for research purposes, it may often be acceptable for various industrial applications.

Another measure of the contamination of the correlogram by multiple scattering is a comparison of the single-scattered intensity at $90^{\circ}$ with the singlescattering cross section:

$$
\sigma_{\text {scatt }}=\pi a^{2} \epsilon_{\text {scatt }} .
$$

This is because multiple scattering is initiated by light that scatters for the first time in any direction whereas only the light scattered for the first time at $90^{\circ}$ arrives at the detectors as singly scattered light. As mentioned above, $I_{\text {single }}\left(90^{\circ}\right)$ is in the ratio 1:32: 454:872 for the 65, 120, 246, and 562-nm PSL spheres considered here. But the single-scattering cross section is in the ratio 1:33:1075:35240. This indicates that multiple scattering is increasingly strong for the 246- and 562-nm PSL spheres, and a residual fewpercent cross-correlation of this amount of multiple scattering can be sizable.

Because the $200-\mu \mathrm{m}$ blocking slit itself should prevent $q$ broadening of the measured correlogram, onebeam cross-correlation experiments were also performed with the index-matching vat absent, but with the remainder of the optical system as in Fig. 1, and with a data collection time of $2 \mathrm{~min}$. The measured sphere radii for this system were found to be generally similar to the results of Fig. 3(a). But the correlograms contained a noticeably larger amount of statistical noise than with the vat present. We believe that the higher noise level is due to lower scattered power coupled into the detector fibers because of the lack of focusing previously provided by the vat.

\section{Discussion}

In Ref. 17 it was found that the one-beam crosscorrelation instrument gave accurate results for the measured particle radius for dilute suspensions of large, optically hard particles. But for strongly turbid suspensions it required long run times and care- 
ful baseline fitting procedures because of the low value of the measured intensity signal-to-baseline ratio. The instrument's high degree of multiplescattering suppression only roughly compensated for the large amount of multiple-scattering intensity admitted by the wide field-of-view detectors. With the improvements in the optical system and sample cell described here, much less multiply scattered light is coupled into the detector fibers, while the ease of alignment of the instrument is retained. As can be seen by the results of Figs. 3(a) and 3(b), data can be acquired much more quickly when working with strongly turbid suspensions.

As a next step in moving the one-beam crosscorrelation instrument toward industrial applications, it should be tested on mixtures of spherical and nonspherical particles of different sizes to determine its particle sizing capability for less than ideal samples. Also, a one-beam cross-correlation instrument with $\theta \approx 165^{\circ}$ (i.e., a cross-correlation backscatter probe) should possess the versatility of autocorrelation backscatter probes ${ }^{4-6}$ while providing improved multiple-scattering suppression and a larger depth of field. The scattering angle $\theta=90^{\circ}$ for the instrument of Fig. 1, although experimentally convenient, represents a relatively unfavorable situation for detecting singly scattered light from large, optically hard particles. This is because as the particle radius increases, the single-scattering light intensity develops a wide minimum centered at $\theta \approx 90^{\circ}$, which is formed by the increasingly rapid falloff of the transmitted intensity for $\theta<90^{\circ}$ and the increasing buildup of the one-internal-reflection and twointernal-reflection intensity ${ }^{26}$ for $\theta>90^{\circ}$. As a result, the single-scattering intensity at $\theta=90^{\circ}$ increases much more slowly and is more easily dominated by multiple scattering than is the case for $\theta \approx$ $165^{\circ}$. Preliminary results for the performance of the one-beam instrument for $\theta$ up to $135^{\circ}$ were given in Ref. 17.

Both particle interaction effects and multiple scattering limit the concentrations of optically hard particles in suspension for which accurate nonintrusive particle sizing measurements can be made using dynamic light-scattering methods, presuming that polydispersity issues have been suitably handled and that flare and optical noise have been eliminated. When $\phi=0.01$, hard-sphere particle interactions cause Eq. (1) to underestimate the radius of the 65-nm PSL spheres by only approximately $1 \%$ and to overestimate the radius of the 246- and 562-nm PSL spheres by approximately $2 \%$. But when $\phi=0.05$, the underestimation and overestimation grow to approximately $5 \%$ and $9 \%$, respectively. Thus even if multiple scattering by the PSL spheres can be suitably suppressed, the volume fraction of the suspension must be less than a few percent if few-percent sizing accuracy is acceptable when using conventional models, such as Eq. (1), for these determinations.

But dynamic light-scattering techniques do not completely eliminate multiple scattering from the measured correlogram. In autocorrelation systems, multiple scattering correlates over the detector's entire field of view, including the region that intersects the laser beam where single scattering greatly dominates, as well as the portion of the field of view that passes through the multiple-scattering halo. In cross-correlation techniques, multiple scattering weakly cross correlates in the overlap region of the beams and the detectors' field of view. The limitation multiple scattering places on the ability of dynamic light-scattering techniques to accurately size particles in suspension depends on the particles' refractive index relative to the liquid, their size, and their concentration as can be seen in Eq. (3), as well as on the geometric configuration of the instrument. For the one-beam cross-correlation instrument described here, the dominant error for sizing PSL spheres in water with volume fraction in the range $0.01<\phi<0.05$ most likely arises from the few-percent residual cross correlation of multiple scattering. But the instrument has succeeded, nonetheless, in extending the utility of the one-beam method by several orders of magnitude.

This research was supported by NASA in the forms of a Summer Student Internship (A. J. Adorjan), an American Society for Engineering Education Summer Faculty Fellowship (T. W. Taylor), NASA grant NCC3-521 (J. A. Lock), and the NASA Advanced Technology Development Program YOF-2567 (P. Tin, W. V. Meyer, and A. E. Smart), and by the Ohio Aerospace Institute in the form of patent disclosures concerning the one-beam instrument.

\section{References}

1. B. J. Berne and R. Pecora, Dynamic Light Scattering (Wiley, New York, 1976), pp. 83-86.

2. P. Stěpánek, "Static and dynamic properties of multiple light scattering," J. Chem. Phys. 99, 6384-6393 (1993).

3. T. Gisler, H. Rüger, S. U. Egelhaaf, J. Tschumi, P. Schurtenberger, and J. Rička, "Mode-selective light scattering: theory versus experimental realization," Appl. Opt. 34, 3546-3553 (1995).

4. H. Wiese and D. Horn, "Single-mode fibers in fiber-optic quasielastic light scattering: a study of the dynamics of concentrated latex dispersions," J. Chem. Phys. 94, 6429-6443 (1991).

5. H. S. Dhadwal, R. R. Ansari, and W. V. Meyer, "A fiber optic probe for particle sizing in concentrated suspensions," Rev. Sci. Instrum. 62, 2963-2968 (1991).

6. R. R. Ansari and K. I. Suh, "Dynamic light scattering particle size measurements in turbid media," in Coherence Domain Optical Methods in Biomedical Science and Clinical Applications II, V. V. Tuchin and J. A. Izatt, eds., Proc. SPIE 3251, 146-156 (1998).

7. G. D. J. Phillies, "Suppression of multiple scattering effects in quasielastic light scattering by homodyne cross-correlation techniques," J. Chem. Phys. 74, 260-262 (1981).

8. G. D. J. Phillies, "Experimental demonstration of multiple scattering suppression in quasielastic light scattering spectroscopy by homodyne coincidence techniques," Phys. Rev. A 24, 1939-1943 (1981).

9. J. K. G. Dhont and C. G. de Kruif, "Scattered light intensity cross-correlation. I. Theory," J. Chem. Phys. 79, 1658-1663 (1983). 
10. H. J. Mos, C. Pathmamanoharan, J. K. G. Dhont, and C. G. de Kruif, "Scattered light intensity cross-correlation. II. Experimental," J. Chem. Phys. 84, 45-49 (1986).

11. M. Drewel, J. Ahrens, and U. Podschus, "Decorrelation of multiple scattering for an arbitrary scattering angle," J. Opt. Soc. Am. A 7, 206-210 (1990).

12. K. Schätzel, M. Drewel, and J. Ahrens, "Suppression of multiple scattering in photon correlation spectroscopy," J. Phys. Condens. Matter 2, SA393-SA398 (1990).

13. K. Schätzel, "Suppression of multiple scattering by photon cross-correlation techniques," J. Mod. Opt. 38, 1849-1865 (1991).

14. L. B. Aberle, P. Hülstede, S. Wiegand, W. Schröer, and W. Staude, "Effective suppression of multiply scattered light in static and dynamic light scattering," Appl. Opt. 37, 6511-6524 (1998).

15. C. Urban and P. Schurtenberger, "Characterization of turbid colloidal suspensions using light scattering techniques combined with cross-correlation methods," J. Colloid Interface Sci. 207, 150-158 (1998).

16. U. Nobbmann, S. W. Jones, and B. J. Ackerson, "Multiple scattering suppression: cross correlation with tilted singlemode fibers," Appl. Opt. 36, 7571-7576 (1997).

17. W. V. Meyer, D. S. Cannell, A. E. Smart, T. W. Taylor, and P. Tin, "Multiple-scattering suppression by cross correlation," Appl. Opt. 36, 7551-7558 (1997).

18. J. A. Lock, "Role of multiple scattering in cross-correlated light scattering with a single laser beam," Appl. Opt. 36, 7559-7570 (1997).

19. E. Hecht, Optics, 2nd ed. (Addison-Wesley, Reading, Mass., 1987), p. 532.

20. J. Rička, "Dynamic light scattering with single-mode and multimode receivers," Appl. Opt. 32, 2860-2875 (1993).

21. C. W. J. Beenakker and P. Mazur, "Diffusion of spheres in concentrated suspension: resummation of many-body hydrodynamic interactions," Phys. Lett. A 98, 22-24 (1983).

22. C. W. J. Beenakker and P. Mazur, "Diffusion of spheres in a concentrated suspension II," Physica A 126, 349-370 (1984).

23. P. N. Pusey and R. J. A. Tough, "Particle interactions," in Dynamic Light Scattering: Applications of Photon Correlation Spectroscopy, R. Pecora, ed. (Plenum, New York, 1985), pp. 85-179.

24. N. W. Ashcroft and J. Lekner, "Structure and resistivity of liquid metals," Phys. Rev. 145, 83-90 (1966).

25. W. V. Meyer, A. E. Smart, D. S. Cannell, R. G. Brown, J. A. Lock, and T. W. Taylor, "Multiple scattering suppression with cross-correlation and flare rejection with fiber optic homodyning," paper AIAA 99-0962, presented at the Thirty-Seventh Aerospace Sciences Meeting and Exhibit, Reno, Nev., 11-14 January 1999 (American Institute of Aeronautics and Astronautics, New York, 1999).

26. J. A. Lock, "Contribution of high-order rainbows to the scattering of a Gaussian laser beam by a spherical particle," J. Opt. Soc. Am. A 10, 693-706 (1993). 